\title{
Degradation of Head Direction Cell Activity during Inverted Locomotion
}

\author{
Jeffrey L. Calton and Jeffrey S. Taube \\ Department of Psychological and Brain Sciences, Center for Cognitive Neuroscience, Dartmouth College, Hanover, New Hampshire 03755
}

\begin{abstract}
Head direction (HD) cells in the rat limbic system carry information about the direction the head is pointing in the horizontal plane. Most previous studies of HD functioning have used animals locomoting in an upright position or ascending/descending a vertical wall. In the present study, we recorded HD cell activity from the anterodorsal thalamic nucleus while the animal was locomoting in an upside-down orientation. Rats performed a shuttle-box task requiring them to climb a vertical wall and locomote across the ceiling of the apparatus while inverted to reach an adjoining wall before ascending into the reward compartment. The apparatus was oriented toward the preferred direction of the recorded cell, or the $180^{\circ}$ opposite direction. When the animal was traversing the vertical walls of the apparatus, the HD cells remained directionally tuned as if the walls were an extension of the floor. When the animal was locomoting inverted on the ceiling, however, cells showed a dramatic change in activity. Nearly one-half (47\%) of the recorded cells exhibited no directional specificity during inverted locomotion, despite showing robust directional tuning on the walls before and after inversion. The remaining cells showed significantly degraded measures of directional tuning and random shifts of the preferred direction relative to the floor condition while the animal was inverted. It has previously been suggested that the HD system uses head angular velocity signals from the vestibular system to maintain a consistent representation of allocentric direction. These findings suggest that being in an inverted position causes a distortion of the vestibular signal controlling the HD system.
\end{abstract}

Key words: vestibular; navigation; upside-down; anterodorsal thalamic nucleus; spatial orientation; rat

\section{Introduction}

Head direction (HD) cells, found primarily in the rat limbic system, carry information about the HD of the animal in the horizontal plane (for review, see Sharp et al., 2001a; Taube and Bassett, 2003). Each HD cell is tuned to a specific "preferred direction," and different HD cells have different preferred directions, presumably allowing the combined activity of the population to reflect the perceived directional heading of the animal. HD cells use a number of different cues to maintain a consistent allocentric directional reference including both landmark cues and internal self-movement (idiothetic) cues (Taube et al., 1990; Taube and Burton, 1995). One feature that has received little experimental attention is the influence of body orientation on the activity of HD cells. Stackman et al. (2000) recorded HD cells while rats used a vertically oriented mesh ladder to move between the floor of the apparatus and an elevated ledge. They found that if the animal approached the ladder with its head oriented in the preferred direction of the recorded cell, it continued to fire as the

Received Aug. 25, 2004; revised Jan. 22, 2005; accepted Jan. 23, 2005.

This work was supported by NASA Cooperative Agreement NCC9-58 from the National Space Biomedical Research Institute and by National Institute of Mental Health Grants MH48924 and MH01286. A preliminary report of this research was presented at the 30th Annual Meeting of the Society for Neuroscience, San Diego, CA, 2000. We thank Matthew Tullman for the collection of some data, Jennifer Marcroft for technical assistance, Richard Johnson for the construction of the apparatus, and Dora Angelaki and Larry Snyder for comments on our findings.

Correspondence should be addressed to Dr. Jeffrey S. Taube, Department of Psychological and Brain Sciences, Dartmouth College, 6207 Moore Hall, Hanover, NH 03755. E-mail: Jeffrey.taube@dartmouth.edu.

J. L. Calton's present address: Department of Psychology, California State University, Sacramento, CA 95819.

DOI:10.1523/JNEUROSCI.3511-04.2005

Copyright $\odot 2005$ Society for Neuroscience $\quad$ 0270-6474/05/252420-09\$15.00/0 animal ascended the ladder, but activity diminished to baseline on the return trip as the animal descended in a head-first orientation. Conversely, when the animal approached the ladder in the direction $180^{\circ}$ opposite the preferred direction of the recorded cell, the cell was quiet while the animal ascended the ladder but was active during the descent. This pattern of activity suggests that the HD cells treated the ladder as a vertical extension of the floor (i.e., the cell responded as would be expected if the wall containing the ladder was folded down to enlarge the floor of the recording enclosure). This finding is not surprising if changes in the HD signal are primarily driven by angular velocity information carried by the horizontal semicircular canals (Taube and Bassett, 2003), because the transition between a horizontal and vertical surface requires a transient change in head pitch, but no head turn to the left or right. The change of surfaces leads to a different overall otolith signal but does not affect the signal conveyed by the semicircular canals. Once the animal is in the vertical plane, activation of the horizontal canals occurs as before whenever the animal rotates its head from side to side. However, if this principle is taken further and the animal performs a second rightangle turn into an inverted orientation (hanging upside down from the ceiling), then there will be a conflict between the actual HD and the HD as determined purely by horizontal angular velocity.

In an attempt to characterize how $\mathrm{HD}$ cell activity might change during $0 \mathrm{~g}$, Taube et al. (2004) recorded HD activity during brief periods of weightlessness produced by parabolic flight. During weightlessness, the animal was positioned manually so 


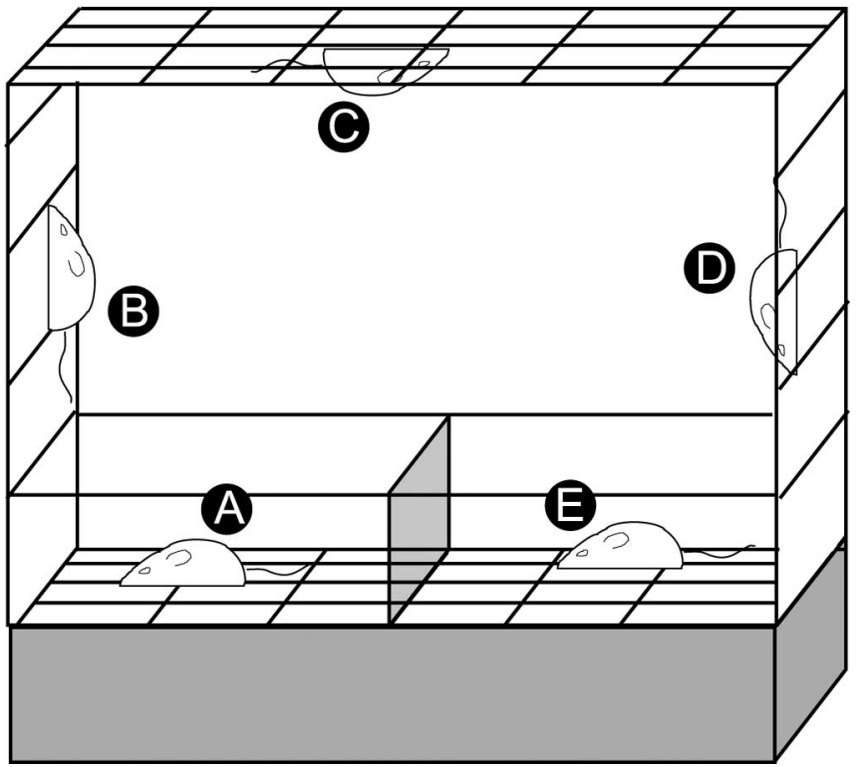

Figure 1. Diagram of recording track and position of a rat over the course of a single trial. The rat begins on one side of the floor surface $(\boldsymbol{A})$. To initiate a trial, the animal approaches and ascends the outer wall $(\boldsymbol{B})$. When the animal reaches the junction between the wall and ceiling, it pitches backward $90^{\circ}$ to move across the ceiling $(C)$ in an upside-down orientation. When the rat reaches the far wall (D), it descends into the reward compartment of the floor $(\boldsymbol{E})$ for a food reward. The current floor compartment then becomes the start compartment, and the animal reverses direction for the next trial.

that it was hanging from the ceiling in an inverted orientation. In this condition, HD cells showed a loss of directional specificity. However, it was not clear whether this loss of tuning was caused by the inverted body position, the $0 \mathrm{~g}$ condition, or because the animal was placed manually in the inverted orientation rather than allowing it to self-locomote into this position. In the same experiment, HD cells maintained directional specificity during the $0 \mathrm{~g}$ condition while the animal locomoted upright on the floor, providing evidence against $0 \mathrm{~g}$ as the sole mechanism of the effect.

In this investigation, we recorded $\mathrm{HD}$ cells from the anterodorsal thalamic nucleus (ADN) when the animal was inverted on the ceiling as described by Taube et al. (2004), but recordings were made in normal gravity, and the animal selflocomoted onto and across the ceiling rather than being passively placed in position. We report that HD cells showed dramatically distorted and often a complete loss of directional tuning during inverted locomotion.

\section{Materials and Methods}

Subjects and behavioral apparatus. Five female Long-Evans rats served as subjects. The weights ranged between 250 and $300 \mathrm{~g}$ at the start of the study. A food-restricted diet was used to maintain the rats at $\sim 85 \%$ of free feeding weight. Water was available ad libitum in the home cage. The animals were housed singly in clear plastic cages in a room maintained on a $12 \mathrm{~h}$ light/dark cycle.

During cell recording, animals traversed the inside of a track formed in the shape of a square ring for a food reward (Fig. 1). Each side was $1.22 \mathrm{~m}$ in length and $0.30 \mathrm{~m}$ wide. The floor, walls, and ceiling of the track were composed of galvanized wire mesh that provided traction for climbing. The bottom surface of the track was enclosed by clear Plexiglas walls and was divided into two equal halves by a clear Plexiglas barrier. Four video cameras were each oriented toward a different surface of the track, allowing the determination of animal location and heading regardless of where the animal was located in the apparatus. The apparatus was mounted on a central pivot point attached to the floor, allowing $360^{\circ}$ rotation of the track relative to the room. Several stable visual landmarks were visible from the track, including a doorway and a black curtain along one wall of the room. The room was dimly illuminated by light from the adjacent doorway.

Behavioral task. Rats were trained to move between the two floor compartments of the track for a food reward (Fig. 1). To successfully complete the task, the animal had to climb the outer wall of the start compartment, locomote across the ceiling in an inverted orientation, and climb down the adjoining wall into the reward compartment where it could consume several small sucrose pellets from a food bowl. The current compartment then became the new start compartment, and the animal was rewarded for repeating the task in the opposite direction. Training the animals in this task required several weeks of shaping. Initially, a wooden platform touching both walls was hung a few inches from the ceiling. Animals were placed on the platform and reinforced for descending the wall to the reward compartment. After mastering this phase, the animals were placed in the start compartment and coaxed to climb up to the platform, cross, and descend to receive a reward. At this point, the wooden platform was removed, and the animal was coaxed across the ceiling while inverted with physical support provided from an experimenter. After much practice, most animals were able to perform the task without support. Within a few more weeks, the animals were able to perform the task in the opposite direction, and then they quickly learned to alternate directions between trials.

Surgical procedures and electrophysiological recording. After completion of behavioral training, each animal received an implantation of a recording electrode array to monitor neural activity during the behavioral task Electrodes were constructed using methods described by Kubie (1984) and consisted of a bundle of 1025 - $\mu \mathrm{m}$-diameter wires that were insulated, except at the tip. The wires were threaded through a stainless steel cannula that was moveable in the dorsal/ventral direction after being fixed to the skull using dental acrylic. Standard surgical procedures were followed for implantation of the recording electrodes (Taube, 1995). Briefly, animals were anesthetized with a ketamine-acepromazine-xylazine mixture. After exposure of the skull, a hole was drilled, and the electrode was implanted just dorsal to the right ADN based on coordinates ( $1.5 \mathrm{~mm}$ posterior to bregma, $1.3 \mathrm{~mm}$ lateral to bregma, and 3.7 $\mathrm{mm}$ below the cortical surface) provided by Paxinos and Watson (1998). After surgery, animals were allowed at least 1 week of recovery before commencement of experimental procedures. All procedures were conducted under an institutionally approved animal care and use protocol.

Electrical signals recorded in the brain were passed through a fieldeffect transistor in a source-follower configuration. Signals were amplified by a factor of 10,000-50,000, bandpass filtered $(300-10,000 \mathrm{~Hz}, 3$ $\mathrm{dB} /$ octave), and sent through a dual window discriminator (BAK Electronics, Mount Airy, MD) for spike discrimination. Screening for HD cells involved examining the electrical signal on each of the 10 implanted wires while the animal foraged in a wooden cylinder for food pellets dropped from above by an automatic food pellet dispenser. If no HD cells were detected, the electrode was advanced $25-50 \mu \mathrm{m}$, and the animal was returned to its home cage. If a HD cell was isolated, the animal was moved to the recording track for data collection.

Recording sessions began by placing the animal in one of the two floor compartments, at which time the animal would typically begin performing the task in alternating directions. During sessions, the position and directional orientation of the rat was monitored using an automated tracking system (Ebtronics, Elmont, NY). This video tracking hardware provided $X$ and $Y$ coordinates $(256 \times 256)$ of red and green lightemitting diodes (LEDs) that were secured $6 \mathrm{~cm}$ apart above the head and back of the animal, respectively. As the animal moved between the different surfaces of the track, an experimenter manually switched the input of the video tracker to the camera oriented toward that particular surface. The firing rate of recorded cells and LED positions were saved onto a personal computer at a rate of $60 \mathrm{~Hz}$. Data analysis was accomplished off-line using custom software (LabView; National Instruments, Austin, TX). The HD of the animal was determined by the relative position of the red and green LEDs. For samples in which the animal was positioned on the floor or hanging from the ceiling, the calculated HD of the animal corresponded to the azimuth that the animal's head was facing using 
Cartesian coordinates, with $0^{\circ}$ and $90^{\circ}$ represented by the directions of east and north, respectively. With respect to the walls, the directional frame of reference was considered to be an extension of the floor [i.e., if an animal approached the eastern wall from the floor while facing $0^{\circ}$ (east) and ascended the wall vertically (without rotating its head to one side or the other), the $\mathrm{HD}$ of the animal was still considered to be $0^{\circ}$ ]. If the animal then moved from the east wall onto the ceiling (without rotating its head to the left or right), the animal was facing $180^{\circ}$. Finally, during the descent of the west wall from the ceiling (without rotating its head), the animal was considered to be once again facing $0^{\circ}$. Because the linear movements of the animals were typically in the direction of the track, the ranges of HDs sampled while actively performing were usually aligned with the long axis of the track. This occurrence was especially problematic on the ceiling and walls, where there were sometimes two narrow ranges of unsampled HDs running at right angles to the long axis of the track. In cases in which the preferred direction of the recorded HD cell was $>45^{\circ}$ from the orientation of the track, the track was rotated to align it with the preferred firing direction of the cell to increase the number of samples in which the animal was facing the preferred direction of the cell. This procedure was not entirely successful, because most cells would rotate somewhat with the long axis of the track (i.e., the track itself exerted partial landmark control of HD activity).

Analysis of HD data. Cellular activity was examined to determine whether a given cell maintained directional tuning while the animal locomoted on each surface. Two methods were used for this analysis. First, firing rate $\times$ HD tuning curves was plotted across each surface for each cell (for examples, see Fig. 3), and the resulting plots were used to subjectively determine whether a cell showed directional modulation of firing activity. The second approach involved performing a Rayleigh analysis (Batschelet, 1981) to determine whether the HDs associated with cellular activity were distributed significantly nonrandomly, as would be expected if a cell was tuned directionally. The critical statistic of the Rayleigh test is the mean vector length, $r$, which varies between 0 and 1 , with higher values indicating that the distribution of directions is clustered. The critical significance level of the mean vector length is determined by the number of observations (e.g., $r_{\text {crit }}=0.173$ for $n=100$ using our $\alpha$ criterion of 0.05 ), and if the $r$ value meets this significance level, the distribution can be said to be nonrandom (Batschelet, 1981). To transform our firing rate $\times \mathrm{HD}$ data into a format appropriate for this test, we divided the $360^{\circ}$ directional range into $1524^{\circ}$ bins and calculated the number of spikes that occurred in each bin. Ideally, this distribution could then be subjected to a grouped Rayleigh test. However, the test assumes that each direction has an equal chance of being sampled (i.e., a spike has an equal chance of occurring in each directional bin), and the raw data do not meet this requirement because some HDs were invariably sampled more than others. To overcome this limitation, the cellular data on each surface were normalized by adjusting the number of spikes occurring in a given directional bin based on what would be expected if it had only been sampled as often as the fewest sampled bin. As an example, assume the fewest sampled bin was sampled 50 times. If another bin was sampled 100 times and during these samples a total of 30 spikes were detected, the number of spikes in this second bin was normalized to 15 spikes. Another assumption of this test is that each bin is sampled at least once. To improve the reliability of the data, we required that each bin be sampled at least 10 times. For cells in which this minimum sampling criterion was not met, the subjective analysis was used exclusively to determine whether the cell maintained directional specificity on a given surface.

To further assess the directional characteristics of recorded cells, the $360^{\circ}$ directional range was divided into $6^{\circ}$ bins, and the following measures were calculated for each cell on each surface: (1) directional information content; (2) preferred direction; (3) peak firing rate; (4) background firing rate; and (5) signal/noise ratio. Directional information content is a measure of how many bits of HD information is conveyed by each spike (Skaggs et al., 1993) and was calculated by the following formula: directional information content $=\sum p_{\mathrm{i}}\left(\lambda_{\mathrm{i}} / \lambda\right) \log _{2}\left(\lambda_{i} / \lambda\right)$, where $p_{\mathrm{i}}$ is the probability that the head pointed in the $i$ th directional bin, $\lambda_{\mathrm{i}}$ is the mean firing rate for bin $i$, and $\lambda$ is the mean firing rate across all directional bins. The preferred direction was the directional bin with the highest average firing rate. The peak firing rate was the firing rate corresponding to the preferred direction. The background firing rate was the average firing rate of the three directional bins located $180^{\circ}$ opposite the preferred direction. The signal/noise ratio was the ratio of the peak firing rate divided by the background firing rate. For the firing rate of a directional bin to be considered valid, it had to have been sampled a minimum of 10 times. This minimum sampling criterion was always met for all directional bins on the floor but was not always met for all bins on the walls and ceiling. However, we do not believe this caused a bias in these measures, because cells were selected for recording and analyses based on the criterion that the preferred directions on the floor tended to line up with the long axis of the track, directions that tended to be sampled heavily on the walls and ceiling. Statistical comparisons of these measures were performed using a Wilcoxon signed rank test.

Histology. At the completion of the experiments, animals were anesthetized deeply, and a small anodal current $(20 \mu \mathrm{A}, 10 \mathrm{~s})$ was passed through one electrode wire to later conduct a Prussian blue reaction. The animals were then perfused transcardially with saline, followed by $10 \%$ formalin in saline. The brains were removed and placed in $10 \%$ formalin for at least $48 \mathrm{~h}$. The brains were then placed in a $10 \%$ formalin solution containing $2 \%$ potassium ferrocyanide for $24 \mathrm{~h}$ and then reimmersed in $10 \%$ formalin $(24 \mathrm{~h}$ ) before being placed in $20 \%$ sucrose for $24 \mathrm{~h}$. They were then frozen, sectioned $(40 \mu \mathrm{m})$ in the coronal plane, stained with cresyl violet, and examined microscopically for localization of the recording sites. All recording electrodes were localized to the ADN.

\section{Results}

A total of $24 \mathrm{HD}$ cells from five animals were monitored while the animal performed the task. Five cells were discarded from analysis because the preferred directions of the cell were sufficiently oriented away from the long axis of the track that the animal did not adequately sample the preferred direction while on surfaces other than the floor, and rotating the track failed to sufficiently improve this sampling. This left a total of 19 cells for additional analysis.

The number of total iterations performed while recording each cell depended on the motivation of the animal to perform the task. In a typical session, the animal would perform 5-10 complete iterations of the task in alternating directions while pausing 0.5-2 min between iterations. As expected from this pattern of behavior, animals typically spent much more time on the floor than on the other three surfaces of the track. Across all recording sessions, animals spent, on average, $17.7 \mathrm{~min}$ on the floor, $2.6 \mathrm{~min}$ on the east wall, $2.6 \mathrm{~min}$ on the west wall, and 3.9 min on the ceiling. On surfaces other than the floor, animals frequently did not sample all directional bins a minimum of 10 times, the number of samples considered necessary for reliable firing rate calculation. Using the more conservative $6^{\circ}$ bin criterion, 11 cells did not meet this requirement for all directional bins on the east wall, for an average inadequately sampled directional range of $45.3 \pm 12.9^{\circ}$. Thirteen cells fit this description on the west wall (average inadequately sampled range, $41.5 \pm 1.0^{\circ}$ ), and nine cells did not meet the minimum sampling requirement for all directional bins while on the ceiling (average inadequately sampled range, $59.3 \pm 7.0^{\circ}$ ). In each of these instances, the inadequately sampled bins were away from the direction of the long axis of the track and were usually divided between two directional ranges falling to either side of the long axis. To provide a sense of the distribution of the less sampled directional bins, the dotted line superimposed on the tuning curves shown in Figure 3 plots the number of samples used to calculate the firing rate plot at each directional bin. Note the pair of lower sampled directional ranges that often occurred on ceiling and wall plots around the $90^{\circ}$ and $270^{\circ}$ directional bins (directions that were orthogonal to the long axis of the track). 


\section{Qualitative description of HD activity during the task}

When the animals were transferred to the recording track, HD cells maintained their directional tuning as the animal explored the floor of the apparatus. When the animal moved from the floor and began to ascend a wall, the cells maintained directional tuning consistent with the walls being an extension of the floor of the apparatus. For instance, if the animal approached a wall in the preferred direction of the cell and subsequently ascended with the head pointed vertically, the cell maintained an elevated firing rate on both the floor and wall. If the animal turned its head while climbing, the cellular activity would, likewise, reflect this change and show decreased firing as though the wall was an extension of the floor. In contrast, the directional tuning appeared much weaker when the animal moved onto the ceiling in an inverted position. If the HD of the animal was oriented toward the preferred direction while climbing a wall (treating the walls as an extension of the floor), then the animal was facing away from the preferred direction while locomoting in an inverted position across the ceiling (assuming a room-centered coordinate system). Cellular activity was monitored audibly through speakers, and if tuning was maintained, we should have noted a dramatic drop in activity at this transition. Although there was always at least some decrease in activity during this transition, cells did not fall silent, as would be expected during normal locomotion in the nonpreferred direction. When the animal moved from the ceiling and began to descend the opposite wall, the cells again were strongly tuned to direction, even when the directionality appeared degraded or nonexistent on the ceiling. Assuming the animal did not turn its head during the descent, cellular activity during this phase was typically high when activity during the ascent of the opposite wall had also been high, once again supporting the conceptualization of the walls as an extension of the floor. When the animal moved from the wall to the floor of the track (the reward compartment of the trial being concluded), the preferred direction of the recorded cell matched the preferred direction shown at the beginning of the trial in the opposite (start) compartment.

When the animal made the reverse trip and moved from the previous goal compartment (now the start box) to the new goal compartment (the previous start box), cell activity was reversed compared with the original trip. Thus, in our hypothetical example of a cell with a preferred firing direction aligned with movement into the new start box, cell activity was low on the return trip as the animal ascended the near wall and low again when it descended the far wall into the new goal box. When locomoting while inverted on the ceiling during this return trip, the animal would be facing the preferred direction of the cell based on room coordinates. In these instances, some cells showed a moderate increase in activity, although this activity was always much less than the activity seen on the other surfaces when facing the preferred direction of the cell. In contrast, other cells showed no obvious change in activity, again illustrating a loss of directional specificity when the animal was inverted on the ceiling.

\section{Subjective and statistical analysis of maintenance of directional tuning}

More systematic analysis of the directional specificity on the different surfaces reinforces the qualitative description. Figure 2 shows a firing rate $\times \mathrm{HD} \times$ time plot for an HD cell during two consecutive task iterations. This particular cell had a preferred direction of $\sim 0^{\circ}$ when the animal was traversing the floor, a feature that can be seen in the top panel as the animal oriented its head toward the east wall before ascending. The maintenance of directional tuning can be seen on the walls by noting the elevated activity when the animal was ascending in the preferred direction (ascending/preferred) relative to activity when the animal was ascending a wall in the nonpreferred direction (ascending/nonpreferred). Likewise, activity was higher when descending in the preferred direction (descending/preferred) than when the animal was descending in the nonpreferred direction (descending/nonpreferred). Remarkably, however, cellular activity was generally unresponsive to HD while the animal traversed the ceiling surface, as can be seen by comparing the ceiling/preferred and ceiling/nonpreferred conditions.

Representative tuning curves for each surface were produced by averaging over all episodes. Several examples of tuning curves are shown in Figure 3. Subjective examination of the tuning curves found that all of the cells (19 of 19) exhibited directional tuning while the animal was on the floor, and all but one of the wall tuning curves (37 of 38) was, likewise, judged to reflect directional tuning. On the ceiling, however, only $52.6 \%$ (10 of 19) of cells had activity subjectively determined to be directionally tuned, and even these 10 cells had attenuated directional tuning on the ceiling as indicated by an increase in background activity and a much less distinct preferred direction compared with the other surfaces (see examples in Fig. 3). In general, we found that there was an increase in background activity at all HDs on the ceiling. Figure 4 shows the distribution of mean vector lengths $(r)$ produced by the Rayleigh analysis across the four surfaces. The Rayleigh analysis of directional tuning was nearly identical to the subjective analysis, with 100\% (19 of 19) of floor and 97.1\% (33 of 34) of wall episodes showing significant directionality but only $52.9 \%$ ( 9 of 17) of ceiling episodes showing significant directional tuning. Among conditions receiving both subjective and Rayleigh analysis, the two measures gave similar conclusions in $92.9 \%$ of cases (65 of 70). Using the subjective analysis, two categories of ceiling conditions were formed, ceiling-directional $(\mathrm{CD} ; n=10)$ (Fig. $3 A)$ and ceiling-nondirectional (CND; $n=9)$ (Fig. $3 B$ ), with additional statistical analyses done separately for these two ceiling conditions. The single nontuned wall episode was excluded from additional analysis. Although two categories of ceiling responses could be defined, note that the distribution of mean vector lengths for the ceiling responses shown in Figure 4 is uniform and not bimodal. In addition, note the increased rate of background firing on ceiling episodes in Figure 3 for both categories of cells.

Table 1 shows the order of occurrence of cells classified as CD or CND during inverted locomotion for each animal. Table 1 demonstrates that cell type was not animal specific, because cells from both categories were found in most animals. In three separate instances, two cells were simultaneously recorded on different wires. In all of these cases, the simultaneously recorded cells showed matching directionality on the ceiling, with two of the pairs showing a loss of directionality and the third pair showing maintenance of directionality. In examining the order of cell type occurrence during recording, it is noteworthy that there was no obvious trend for one cell type to consistently precede the other. However, within each animal, there does appear to be a clustering of cell category. The fact that the order of cell type occurrence differs across animals argues against a simple experiencedependent or anatomical explanation for the clustering. Furthermore, the data suggest that the directionality shown on the ceiling by an HD cell was more likely determined by the state of the animal, rather than a physiological distinction between $\mathrm{CD}$ and CND HD cells.

Cells that were categorized as CND typically reestablished di- 

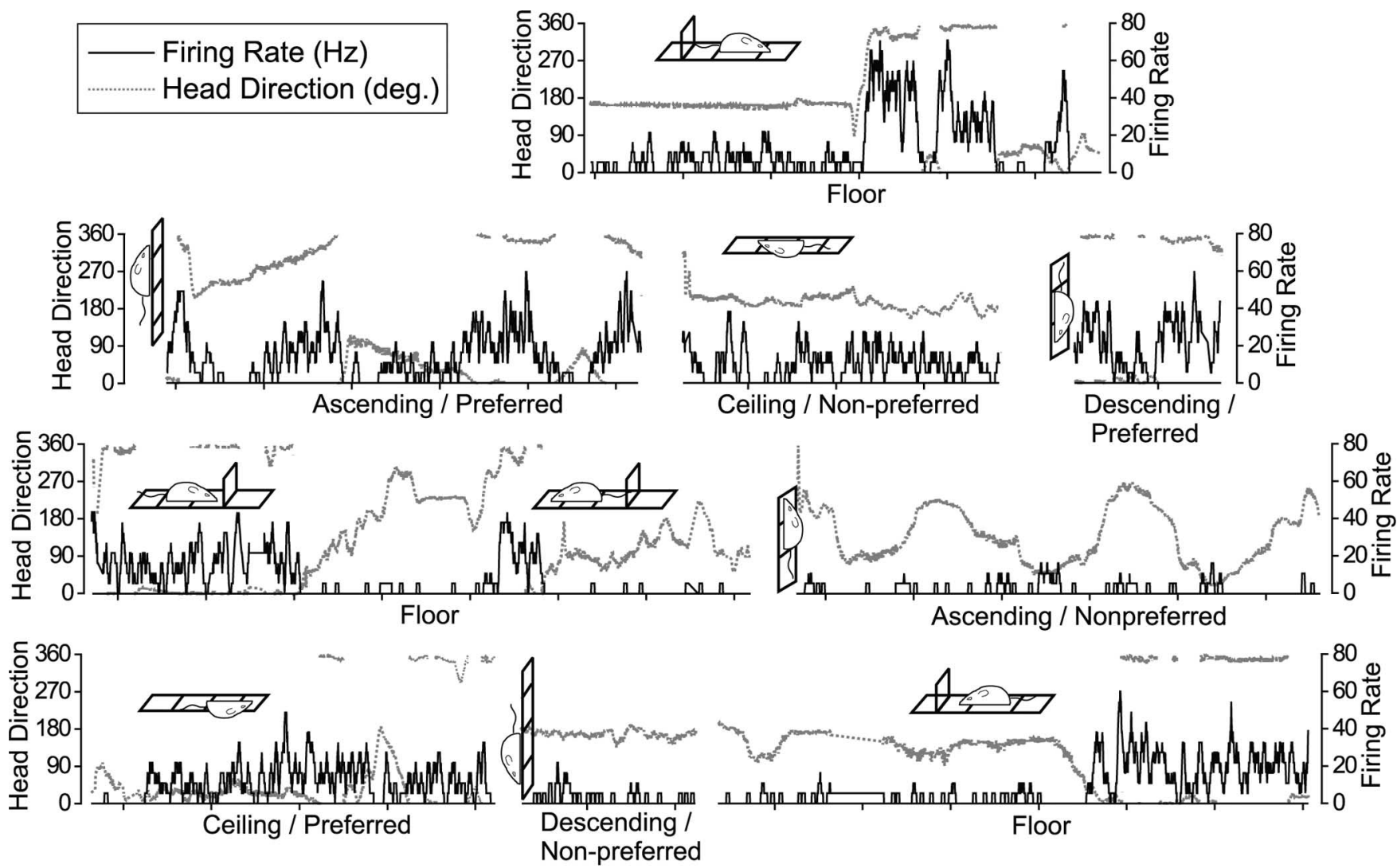

Figure 2. Firing rate $\times H D \times$ time plot for two consecutive trials. Time is represented on the abscissa with the distance between tic marks indicating $5 \mathrm{~s}$. The $H D$ of the animal is indicated as a dotted gray line using the left ordinate, whereas the firing rate of the recorded cell is plotted as the black line on the right ordinate. The label under each panel indicates the current surface being traversed and the general direction of locomotion relative to the preferred direction of the cell. This cell had a preferred direction of $\sim 0^{\circ}$ (east). Note the influence of $\mathrm{HD}$ on the firing rate on the floor and when the animal ascends and descends the walls (compare panels labeled preferred and nonpreferred). In contrast, when the animal was locomoting on the ceiling, the firing rate of the cell was generally not affected by HD. The firing rate trace was smoothed by averaging each value across five points forward and backward in time. The HD trace was not smoothed. deg., Degree.

rectional control when the animal moved from the ceiling onto the wall and began to descend. Note that Figure 3 is not suitable for showing this, because each wall panel includes both ascending and descending data. This feature is illustrated for an example cell in Figure 5 by sorting the wall data based on whether the animal was ascending toward or descending from the ceiling. Similar to the cell shown in the Fig. 5, all but one of the eight CND cells subjected to this analysis appeared to reestablish directional control as the animal descended the wall from the ceiling (one cell was excluded from this analysis because of insufficient directional sampling while the animal was descending). Because of the imprecision inherent in the experimenter-controlled manual transition from the ceiling to wall camera, as well as knowing when to define the transition point (i.e., is it when the head, forelimb, or hindlimb position shifts to the next plane?), it was not possible to determine exactly the time course of this reestablishment of directional tuning on the descent. However, our subjective perceptions during data collection and examination of the firing rate $X$ $\mathrm{HD} \times$ time plots led us to conclude that this shift occurred quite rapidly if not instantaneously. This can be seen in Fig. 2 by noting the rapid increase in activity of the panels labeled "descending/ preferred" and the rapid decrease in activity of the panels labeled "descending/nonpreferred."

\section{Other directional characteristics}

The maintenance of directional tuning when the animal moved from the floor to another surface does not necessarily indicate that the cell maintained the same preferred direction on the dif- ferent surfaces. To calculate the shift in preferred direction between the floor and other surfaces, the preferred direction on each surface was subtracted from the preferred direction on the floor. Figure 6 presents these deviation scores, with scores around zero indicating those sessions in which the preferred direction did not appreciably shift between the floor and the other surface. As indicated in Fig. 6, the preferred directions of the cells while the animal traversed the walls were similar to those of the floor (shift deviations centered around $0^{\circ}$ ). A Rayleigh test showed both of the wall distributions to be significantly different from random (mean vector lengths, $r=0.79$ and 0.96 ; mean vectors, $m=-1.4^{\circ}$ and $\left.-4.4^{\circ} ; p<0.05\right)$. On the other hand, the distribution of angular deviations between floor and ceiling preferred directions for $\mathrm{CD}$ cells was not significantly different from ran$\operatorname{dom}\left(r=0.52 ; m=52.1^{\circ} ; p>0.05\right)$, indicating that the preferred direction tended to shift randomly even in instances when the HD cell maintained directional modulation on the ceiling. The bottom right panel of Figure 6 compares the preferred directions of the east and west floor compartments and illustrates strong directional stability despite fluctuations in the reward status of a compartment and the loss of directionality on the ceiling when traversing between the compartments $\left(r=0.97 ; m=1.6^{\circ} ; p<\right.$ $0.05)$.

Figure 7 presents mean values for directional information content, peak firing rate, background firing rate, and signal/noise ratio for each of the four surfaces. Only cells that were categorized as being directional on a particular surface were included in these analyses. The floor and wall surfaces were not significantly differ- 

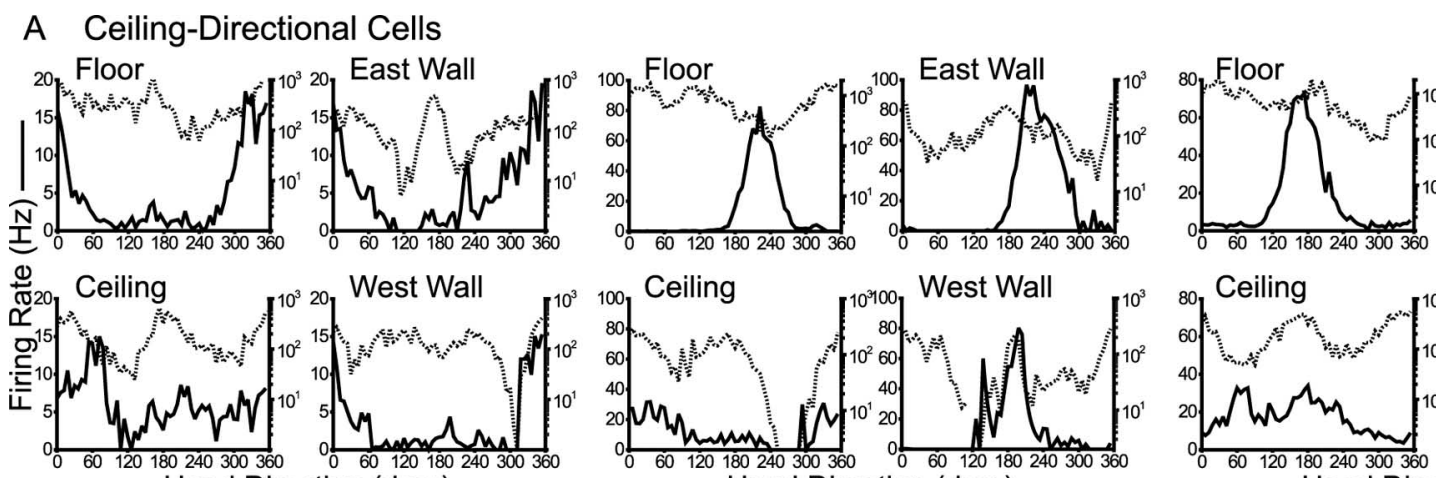

Head Direction (deg.)

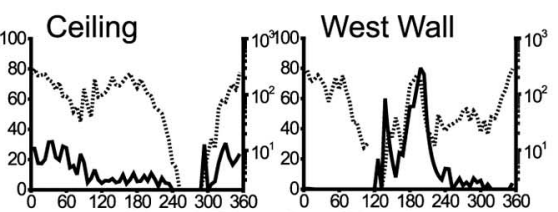

Head Direction (deg.)

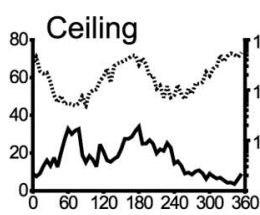

Head Direction (deg.)
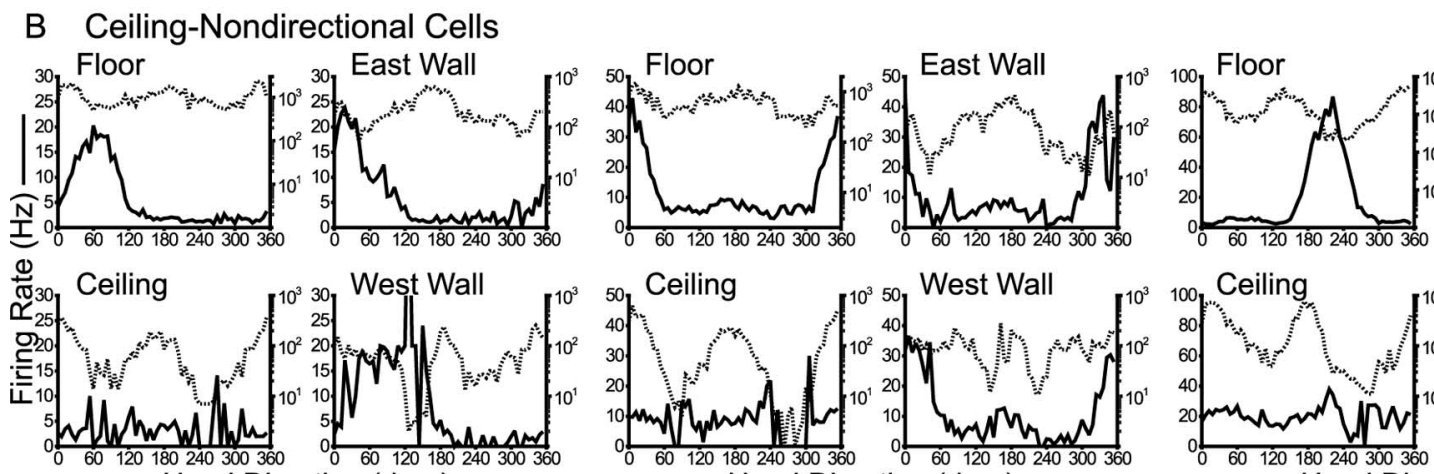

Head Direction (deg.)
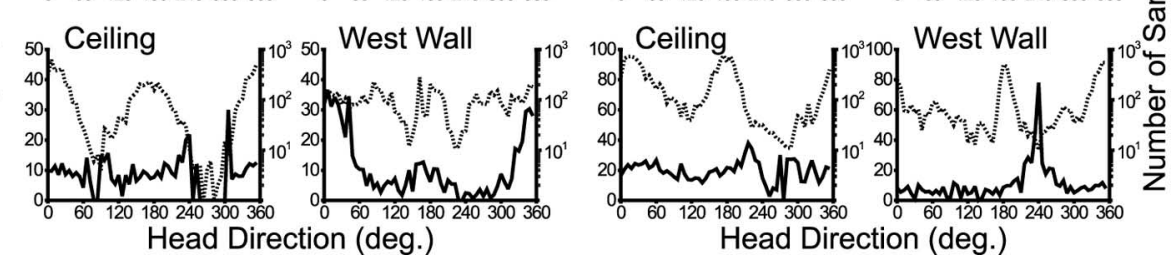

Figure 3. Representative firing rate $\times H D$ tuning curves across each of the four surfaces. $H D$ is shown on the abscissa, the firing rate is plotted as a solid line relative to the left ordinate, and the number of samples at each directional bin is plotted as a dotted line relative to the right ordinate using a logarithmic scale. $A$, Tuning curves from three different $H D$ cells categorized as $C D$. Note the present but degraded directional specificity while the animal was traversing the ceiling in an inverted orientation. $\boldsymbol{B}$, Tuning curves from three HD cells categorized as CND. Note the absence of directional specificity while the animal was traversing the ceiling. Plots are constructed using $6^{\circ}$ directional bins. deg., Degree.

Directional Cells $\square$ Nondirectional Cells
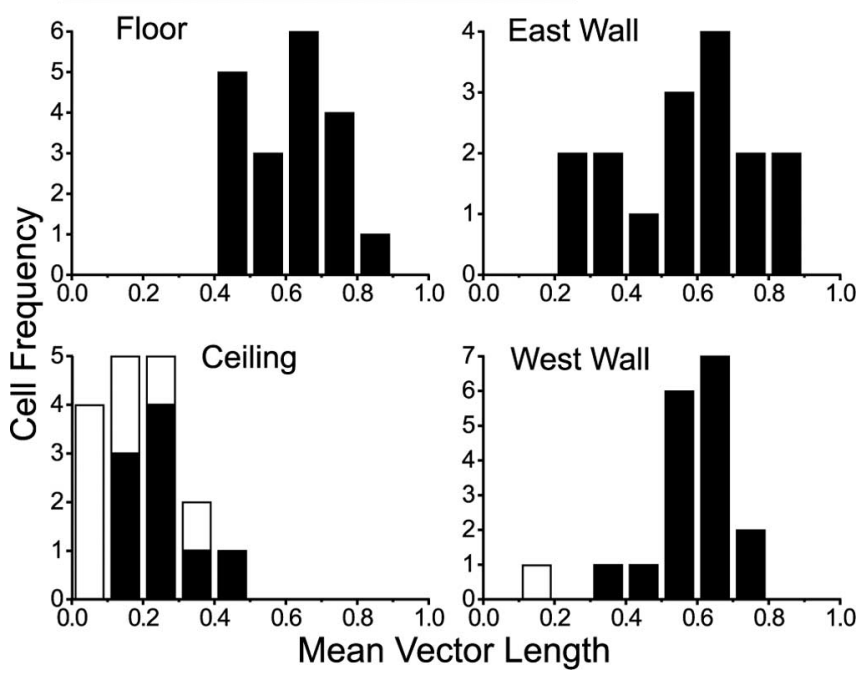

Figure 4. Frequency distributions of mean vector lengths obtained from Rayleigh analysis of directional tuning. Mean vector length is a measure of directional specificity, with increasing values indicating cellular activity dependent on the HD of the animal. The filled bars indicate cells showing significant $(p<0.05)$ directional specificity on that surface, whereas the open bars indicate cells failing to show directional specificity on that surface.

ent on any of the four measures $(p>0.05)$. Ceiling episodes were significantly lower than floor episodes on directional information content $\left(V_{(10)}=55 ; p<0.01\right)$, significantly lower on peak firing rate $\left(V_{(10)}=55 ; p<0.01\right)$, significantly higher on background firing rate $\left(V_{(8)}=2 ; p<0.05\right)$, and significantly lower on
Table 1. Occurrence of CD and CND HD cells during inverted locomotion in each animal

\begin{tabular}{lllllll}
\hline Rat 1 & CD & CD & CD & CD & CND & CND \\
Rat2 & CND & CND & CD & & & \\
Rat3 & CD & & & & & \\
Rat 4 & CND & CND & CND & CD & & \\
Rat5 & CD & CD & CD & CND & CND & \\
\hline
\end{tabular}

For each rat, cells are shown in chronological order of recording. Underlines indicate the three pairs of cells that were recorded simultaneously.

the signal/noise ratio $\left(V_{(8)}=35 ; p<0.05\right)$. Together, these statistical measures support the subjective observation that even in the cases in which some semblance of ceiling tuning was maintained, the directional signal was dramatically degraded while the animal traversed the ceiling.

\section{Discussion}

The discovery of cells carrying information about the HD of the animal (HD cells) and cells carrying information about the location of the animal (place cells) has greatly influenced theorists interested in the neural mechanisms of navigation. Although these two cell types share many important features such as control by both external landmarks and internal idiothetic cues, an important difference is the degree to which spatially relevant activity is maintained across a variety of situations. Place cells exhibiting location-specific activity in a given environment or behavioral context will commonly become silent if the situation changes (O'Keefe and Conway, 1978; Kubie and Ranck, 1983). HD cells, on the other hand, typically maintain directionally specific responding across different environments or behavioral tasks (Taube et al., 1990; Golob et al., 2001). Although the pre- 

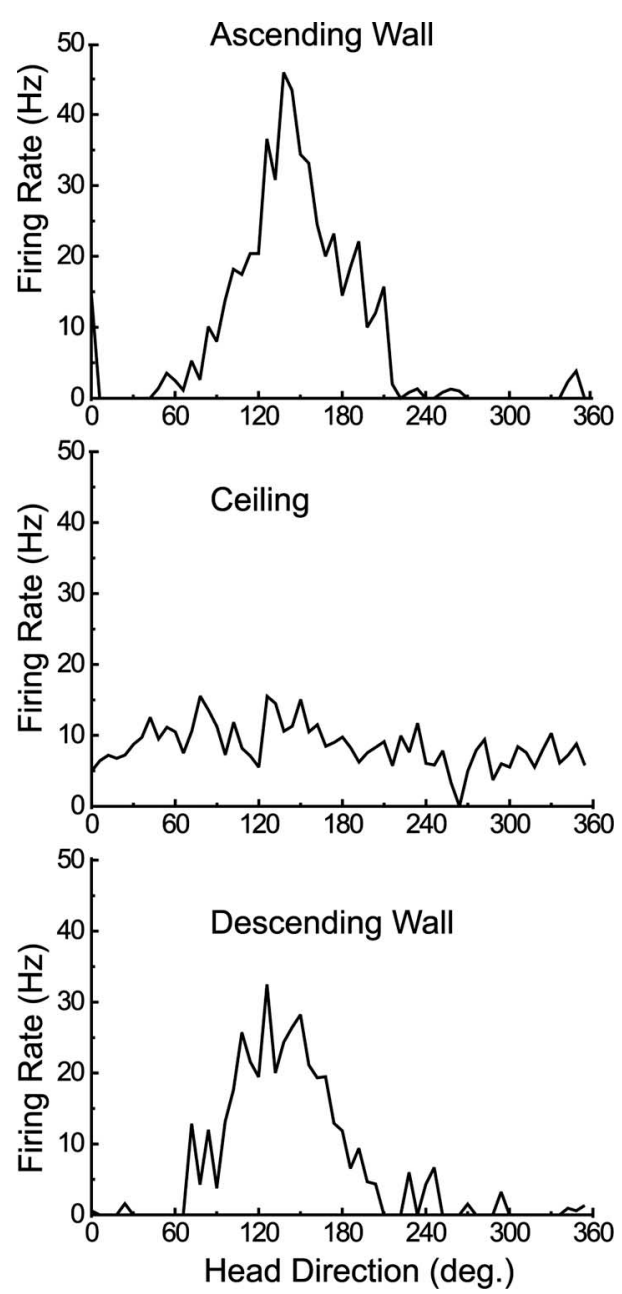

Figure 5. Firing rate $\times H D$ tuning curve of a representative $H D$ cell plotted as a function of whether the animal was ascending a wall, locomoting inverted across the ceiling, or descending a wall. Note that the directional specificity is maintained when the animal descended, although the cell was nondirectional on the ceiling. deg., Degree.

ferred direction of HD cells will often shift if the animal is placed in an environment in the absence of polarizing cues, no previous study has shown an instance in intact animals under normal gravity in which HD cells lose directional specificity during freely moving locomotion. Here we show that the directional specificity of HD cells was lost or dramatically distorted when the animal locomoted in an upside-down body orientation.

A number of explanations might account for this finding, including the possibility that the effect is an artifact of some feature of the inverted recording procedure. For instance, the number of samples collected while the animal was traversing the ceiling and walls was much less than collected in a typical HD cell experiment, and so the apparent lack of directional tuning on the ceiling might perhaps be attributable to insensitivity because of the fewer number of samples in each directional bin. We reject this possibility because the number of samples collected on the ceiling was greater than the number of samples on the walls (an average of $3.9 \mathrm{~min}$ on the ceiling vs $2.6 \mathrm{~min}$ on the walls) and directional tuning was preserved in virtually every instance on the walls. Similarly, the animals often sampled an incomplete range of HDs while inverted, particularly for bins corresponding to directions orthogonal to the longitudinal axis of the track, bringing up the possibility that some cells remained tuned, but their preferred directions fell in unsampled bins. The fact that CD cells

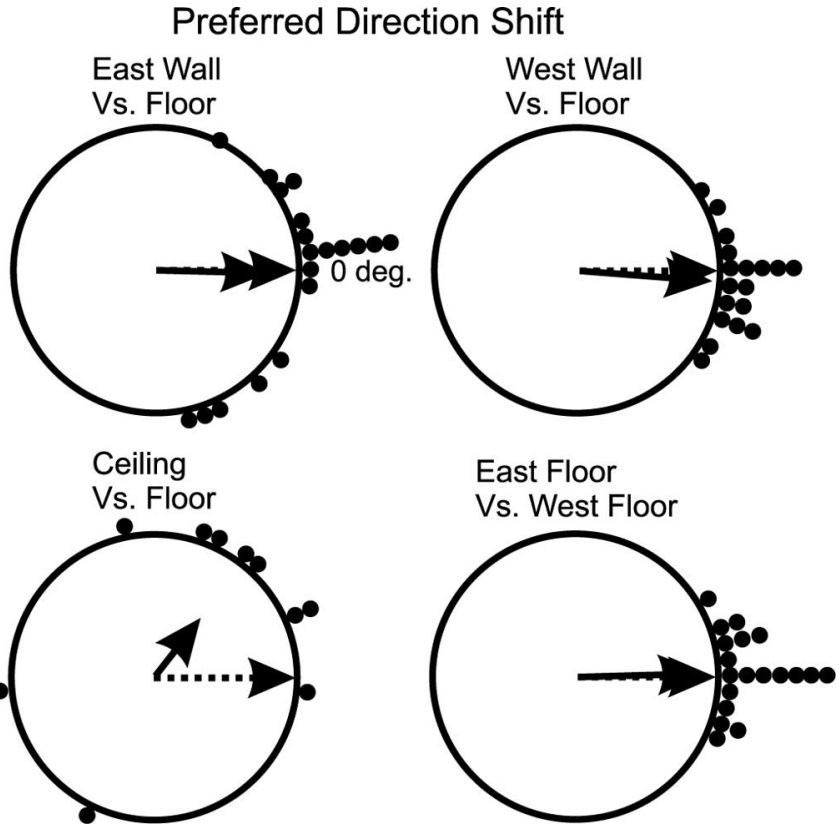

Figure 6. Scatter diagrams showing the amount of angular shift of preferred directions between different surfaces. While traversing the walls, cells tended to show preferred directions similar to the floor (top row). In contrast, preferred directions tended to shift randomly while the animal was locomoting in an inverted position (bottom left panel). Cells showed stable tuning when the east and west compartments of the floor surface are compared (bottom right panel). The angular position of each filled circle represents the shift of preferred direction for individual cells between the two surfaces. For each comparison, only angular shifts from the cells showing maintenance of directional tuning on both surfaces are shown. The dotted arrow denotes an angular shift of zero, and the solid arrow denotes the observed mean vector angle. The length of the solid arrow denotes the mean vector length; a length of 1.0 (no variability in shift scores) is represented by a vector spanning the radius of the circle.

often showed random shifts potentially away from the long axis of the track lends credibility to this explanation. However, because undersampled regions typically occurred in two very narrow ranges lying orthogonal to the track (dotted lines of Fig. 3), we do not believe there was much opportunity to miss directional cells even if they had experienced a dramatically shifted preferred direction while inverted, because these narrow ranges were considerably smaller than the typical $90^{\circ}$ firing range of $\mathrm{HD}$ cells, and the directional bins surrounding this undersampled range gave little indication of direction-specific firing.

\section{Underlying mechanisms for the loss of directional tuning}

Assuming our finding of loss of ceiling directionality is valid, several mechanisms can be postulated. In considering the distinction between those cells categorized as directional or nondirectional on the ceiling, the concordance between simultaneously recorded cells leads us to conclude that this distinction has more to do with the current state of the animal than a physiological difference between the two categories. Although the directionality of simultaneously recorded cells and the presence of cell type clustering within individual animals opens the possibility of an anatomical segregation between cell type, for this segregation to occur, it would have to be more complex than a simple dorsal versus ventral distribution, because the two cell types were found in different orders across different animals. Finally, we emphasize that even those cells categorized as "directional" during inverted locomotion showed much less directional specificity (as exemplified by the attenuated signal/noise ratio and lower directional information content) than when the animals were traversing 

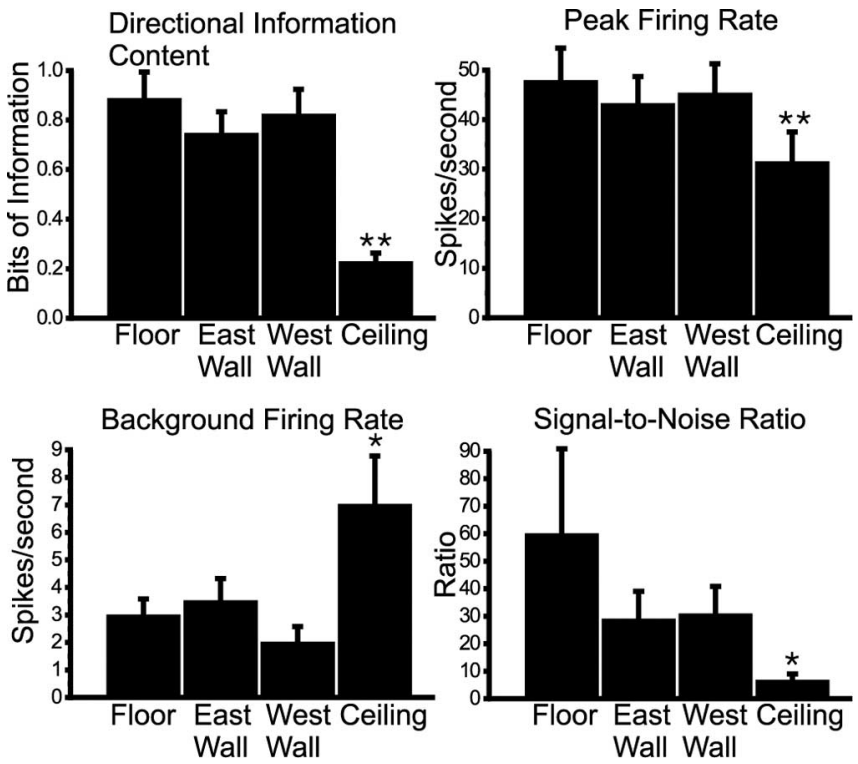

Figure 7. Mean values ( \pm SEM) of directional characteristics across each of the four surfaces. For each statistic, the directional characteristic was degraded on the ceiling relative to the other three surfaces. Means from each surface were calculated only from cells categorized as directional on that surface. One and two cells had background firing rates of zero on the east and west walls, respectively. Because a zero background rate for these cells would have resulted in an infinite signal/noise ratio, these cells were assigned background values equal to the lowest values within their respective condition for the purpose of calculating the signal/noise ratio. Comparisons were relative to the floor condition using the Wilcoxon signed rank test $\left({ }^{*} p<\right.$ $\left.0.05 ;{ }^{* *} p<0.01\right)$.

other surfaces. Indeed, the distribution of Rayleigh scores obtained during inverted locomotion (Fig. 4) was more uniform than bimodal, suggesting a gradient of directionality loss rather than an all-or-none deficit.

Perhaps the signal from the HD system reflects the animal's perceived directional heading in the environment and our animals were disoriented while inverted on the ceiling. To date, there has been little study on how HD cells respond when an animal is disoriented. Preliminary experiments have shown that HD cells lose their directional tuning when animals are spun continuously or back-and-forth on a turntable for tens of seconds (Steven and Taube, 2002). However, in the present experiment, the animals performed quite well in the task and appeared "to know" which direction to proceed to reach the reward compartment, arguing against disorientation as the mechanism of the effect. Correct performance is not surprising, given the extensive training the animals received before cell recording. Indeed, our results indicate a dissociation between the animal's behavior on the ceiling and the information provided by ADN HD cells. Although the cells frequently lost their directional tuning on the ceiling, the animals were still capable of proceeding to the reward compartment accurately, suggesting that mechanisms other than the HD cell system were guiding spatial behavior. This dissociation has been observed with HD cells under other conditions (Golob et al., 2001; Muir and Taube, 2002). Finally, there is no evidence that HD cells lose their directional specificity when an animal is exposed to a situation requiring low navigational demand. For example, robust directional tuning is obtained from HD cells when an animal simply forages for food rewards inside a circular enclosure.

HD cells use both idiothetic (self-movement) cues and external landmark information to maintain a stable directional signal. Vestibular information is especially important because destruc- tion or temporary inactivation of the vestibular labyrinth eliminates HD activity (Stackman and Taube, 1997; Stackman et al., 2002). It has been suggested that vestibular information converges on the HD system via a subcortical stream originating from the medial vestibular nuclei (Taube and Bassett, 2003). This view is supported by the discovery of angular head velocity signals in the dorsal tegmental nucleus (Bassett and Taube, 2001; Sharp et al., 2001b), a structure that receives projections from the vestibular nuclei via the nucleus prepositus and projects to the lateral mammillary nucleus, the first area in the proposed pathway in which HD cells are the predominant cell type.

Perhaps being in an inverted orientation results in distortion of vestibular functioning and subsequent distortion of the HD signal. The semicircular canals of the inner ear are the primary vestibular source of detecting rotational acceleration of the head, whereas the otolith organs respond to inertia and gravity to detect translational movements and head tilt from the vertical axis. Although we know of no evidence that the functioning of the canals would be affected by the orientation of the head relative to gravity, the otolith signal has been shown to be distorted at nonupright orientations (Walsh, 1960; Plotnik et al., 1999), and the stabilization of head and eye position in space is likely a function of vestibular information converging from both of these sources. Indeed, many neurons of the medial vestibular nuclei, the proposed source of vestibular information reaching the HD system, seem to integrate both canal and otolith signals (Bush et al., 1993).

The horizontal and vertical vestibulo-ocular reflexes (VORs) serve to stabilize eye position during angular head movements and, although these reflexes are primarily mediated by the semicircular canals, some otolith activation also occurs for some angular movements. These reflexes have also shown distortion during unusual head orientations relative to the gravity vector. Angelaki and Hess (1994) found abnormal post-rotational nystagmus of the VOR when rotating monkeys in the dark toward orientations that approached upside-down. Working with rats, Brettler et al. (2000) sought to determine the otolith contribution to the horizontal and vertical VOR by giving angular rotations from a variety of body orientations relative to gravity. This study found that angular rotations that activated both the canals and otoliths (e.g., a roll from an upright position) elicited more accurate VORs than rotations that activated the canals alone (e.g., yaw rotations to the left or right from an upright position). Especially relevant to the present findings, rats subjected to rolls while starting from an inverted orientation showed a distorted vertical VOR, with vertical eye movements occurring in the same direction as the roll (an anticompensatory VOR) for low-frequency accelerations and large phase errors during all frequencies. Although the authors did not test the horizontal VOR from an inverted position, clearly the potential exists for a similar distortion. In summary, although current views primarily attribute the horizontal semicircular canals with providing the necessary vestibular signal for driving the HD system, there is potentially an influence from the otoliths, and the distortion of this system during an inverted body orientation is a possible explanation for the distorted HD cell responses during inverted locomotion in our animals.

\section{Implications for models of HD functioning}

Models of HD functioning often rely on the use of an "attractor" network, in which HD cells with overlapping preferred directions share excitatory connections and cells with greatly dissimilar preferred directions inhibit each other (Skaggs et al., 1995; Sharp et 
al., 1996; Zhang, 1996; Goodridge and Touretzky, 2000). This results in a self-sustaining "hill" of activity in the HD network centered on the instantaneous HD of the animal. Stimuli such as vestibular, proprioceptive, efferent copy, or landmark cues are thought to move the activity hill as the animal changes HD. The loss of directional tuning while inverted and the subsequent reestablishment of the signal has potential implications for this type of model. Assuming the loss of tuning we observed is attributable to a disturbance in the vestibular signal, and assuming the vestibular signal is an important external stimulus for directing the activity hill within the attractor network, such models could predict that the activity hill would become stationary as the animal moves from the wall to the ceiling. This occurrence would have predicted a reversal of preferred directions while inverted, because ascension in the nonpreferred direction preceded movement across the ceiling in the preferred direction and, conversely, ascension in the preferred direction preceded inverted movement in the nonpreferred direction. Our findings did not support this view; however, it is notable that vestibular lesions themselves also fail to support this prediction of attractor models (Stackman and Taube, 1997; Stackman et al., 2002). Given the fact that we found a complete absence of tuning in many cells during inversion, our results suggest a more basic disruption in the generation of the signal, rather than an inability to move an intact attractor hill during locomotion.

Cells regained directionality quickly once the animal moved from the ceiling onto the wall and began to descend. This reestablishment of tuning could have occurred because of resumption of normal vestibular functioning, the sudden availability of nonvestibular cues such as visual landmarks, or a combination of these factors. It would be interesting to remove the visual landmarks on some trials to determine whether they are necessary for the reestablishment of tuning. Such a manipulation would provide evidence for or against the view that the directional signal (activity hill) has been completely eliminated rather than simply masked by vestibular dysfunction and requires the presence of a calibrating landmark to be reestablished.

\section{References}

Angelaki DE, Hess BJ (1994) Inertial representation of angular motion in the vestibular system of rhesus monkeys. I. Vestibuloocular reflex. J Neurophysiol 71:1222-1249.

Bassett JP, Taube JS (2001) Neural correlates for angular head velocity in the rat dorsal tegmental nucleus. J Neurosci 21:5740-5751.

Batschelet E (1981) Circular statistics in biology. London: Academic.

Brettler SC, Rude SA, Quinn KJ, Killian JE, Schweitzer C, Baker JF (2000) The effect of gravity on the horizontal and vertical vestibule-ocular reflex in the rat. Exp Brain Res 132:434-444.

Bush GA, Perachio AA, Angelaki DE (1993) Encoding of head acceleration in vestibular neurons. I. Spatiotemporal response properties to linear acceleration. J Neurophysiol 69:2039-2055.

Golob EJ, Stackman RW, Wong AC, Taube JS (2001) On the behavioral significance of head direction cell: neural and behavioral dynamics during spatial memory tasks. Behav Neurosci 115:285-304.

Goodridge JP, Touretzky DS (2000) Modeling attractor deformation in the rodent head-direction system. J Neurophysiol 83:3402-3410.
Kubie JL (1984) A driveable bundle of microwires for collecting single-unit data from freely-moving rats. Physiol Behav 32:115-118.

Kubie JL, Ranck Jr JB (1983) Sensory-behavioral correlates in individual hippocampus neurons in three situations: space and context. In: Neurobiology of the hippocampus (Seifert W, ed), pp 433-447. New York: Academic.

Muir GM, Taube JS (2002) The neural correlates of spatial navigation and performance: do head direction and place cells guide behavior? Behav Cogn Neurosci Rev 1:297-317.

O'Keefe J, Conway DH (1978) Hippocampal place units in the freely moving rat: why they fire where they fire. Exp Brain Res 31:573-590.

Paxinos G, Watson C (1998) The rat brain in stereotaxic coordinates. San Diego: Academic.

Plotnik M, Freeman S, Sohmer H, Elidan J (1999) The effect of head orientation on the vestibular evoked potentials to linear acceleration impulses in rats. Am J Otol 20:735-740.

Sharp PE, Blair HT, Brown M (1996) Neural network modeling of the hippocampal formation spatial signals and their possible role in navigation: a modular approach. Hippocampus 6:720-734.

Sharp PE, Blair HT, Cho J (2001a) The anatomical and computational basis of the rat head direction cell signal. Trends Neurosci 24:289-294.

Sharp PE, Tinkelman A, Cho J (2001b) Angular velocity and head direction signals recorded from the dorsal tegmental nucleus of Gudden in the rat: implications for path integration in the head direction cell circuit. Behav Neurosci 115:571-588.

Skaggs WE, McNaughton BL, Gothard KM, Markus EJ (1993) An information-theoretic approach to deciphering the hippocampal code. In: Advances in neural information processing systems, Vol 5 (Hanson SJ, Cowan JD, Giles CL, eds), pp 1030-1037. San Mateo, CA: Morgan Kaufmann.

Skaggs WE, Knierim JJ, Kudrimoti HS, McNaughton BL (1995) A model of the neural basis of the rat's sense of direction. In: Advances in neural information processing systems (Tesauro G, Touretzky DS, Leen TK, eds), pp 173-180. Cambridge, MA: MIT.

Stackman RW, Taube JS (1997) Firing properties of head direction cells in rat anterior thalamic neurons: dependence upon vestibular input. J Neurosci 17:4349-4358.

Stackman RW, Tullman ML, Taube JS (2000) Maintenance of rat head direction cell firing during locomotion in the vertical plane. J Neurophysiol 83:393-405.

Stackman RW, Clark AS, Taube JS (2002) Hippocampal spatial representations require vestibular input. Hippocampus 12:291-303.

Steven MS, Taube JS (2002) Head direction cell discharge during periods of disorientation. Soc Neurosci Abstr 28:584.3.

Taube JS (1995) Head direction cells recorded in the anterior thalamic nuclei of freely moving rats. J Neurosci 15:70-86.

Taube JS, Bassett JP (2003) Persistent neural activity in head direction cells. Cereb Cortex 13:1162-1172.

Taube JS, Burton HL (1995) Head direction cell activity monitored in a novel environment and during a cue conflict situation. J Neurophysiol 74:1953-1971.

Taube JS, Muller RU, Ranck Jr JB (1990) Head-direction cells recorded from the postsubiculum in freely moving rats. II. Effects of environmental manipulations. J Neurosci 10:436-447.

Taube JS, Stackman RW, Calton JL, Oman CM (2004) Rat head direction cell responses in zero-gravity parabolic flight. J Neurophysiol 92:2887-2997.

Walsh EG (1960) Perception of linear motion following unilateral labyrinthectomy: variation of threshold according to the orientation of the head. J Physiol (Lond) 153:350-357.

Zhang K (1996) Representation of spatial orientation by the intrinsic dynamics of the head direction cell ensemble. J Neurosci 16:2112-2126. 